\title{
The Influence of Crack Parameters on the Static and Dynamic Frequency of Wind Turbine Blades
}

\author{
Yonggang LIU ${ }^{1, a}$, Yazhou MAO ${ }^{2, b}$, Dongying $\mathrm{LI}^{3, \mathrm{c}}$ \\ ${ }^{1}$ School of Mechatronics Engineering, Henan University of Science and \\ Technology,Luoyang,471003,China \\ ${ }^{2}$ School of Mechatronics Engineering,Henan University of Science and \\ Technology,Luoyang,471003,China \\ ${ }^{3}$ School of Mechatronics Engineering,Henan University of Science and \\ Technology,Luoyang,471003,China \\ aemail:martin_lyg@163.com, ${ }^{\text {aemail:myzlcc@163.com, }{ }^{\mathrm{C} e m a i l}: I d d y y l b @ 163 . c o m ~}$
}

Keywords: blade crack;static and dynamic frequency;crack position;crack depth

\begin{abstract}
In this paper, the static and dynamic equations of the blade are derived, the comparison between analytical method and finite element analysis results. Finding the error of the two methods is less than $1 \%$. The influence of the distribution characteristics of the blade crack on the static and dynamic frequency was studied by using ANSYS software. The result shows that the influence of crack direction on frequency is similar, the effect of the different speed on the dynamic frequency of the blade is not the same.With the change of crack position, the dynamic and static frequency of the blade decreases and then increases. The deeper the crack depth, the lower the dynamic and static frequency of the blade.
\end{abstract}

\section{Introduction}

Wind turbine blade carries the main task of energy conversion, which is an important component of wind energy conversion. The initiation and cessation of the frequent periodicity and randomness of the blades are the root causes of the failure of the cracks. The natural frequency of the blade is affected by the depth and position of the crack.

The cracked blade natural frequency change is caused by blade stiffness or quality changes. The change of the stiffness or quality of the structure will lead to different degree of detuning.The detuning of the blade brings great threat to the safe operation of the wind turbine.The literature [1], the effect of crack parameters on the frequency steering characteristics of aero engine blade was analyzed. The literature [2], by introducing the stress stiffening of the blade and the effect of the softening of the blade on the natural frequency of the blade The literature [3], the low-order vibration characteristics of leaf blades were investigated by the variation of crack and depth in the inlet and outlet of cracked blades.

In this paper, ANSYS software is used to study the influence of different cracks on the position and depth of the blade on the first order static and dynamic frequency of wind turbine blades.

\section{Numerical Analysis}

In this paper, the fixed root of blades equivalent torsion free and equal section into a uniform cantilever beam structure using Rayleigh method for dynamic frequency formula. Assuming that there exists a modal function satisfying the boundary, the fundamental frequency can be solved [4] . According to the Rayleigh energy method of bending beam energy.

$M=E I \frac{\partial^{2} y}{\partial x^{2}} d x$

$U_{\max }=\int_{0}^{l} \frac{M^{2}}{2 E I} d x=\frac{1}{2} \int_{0}^{l} E I\left(\frac{\partial^{2} y_{0}}{\partial x^{2}}\right)^{2} d x$

In the type: $E$ represents elastic modulus, $I$ represents moment of inertia of the section, $Y_{0}$ 
expressed vibration type function, $l$ represents the height of the blade.

The kinetic energy of the cantilever beam:

$T_{\max }=\frac{1}{2} m y_{0} \Omega \sin \Omega t=\frac{1}{2} \int_{0}^{l} \rho A \Omega^{2} y_{0}^{2} d x$

In the type: $\rho$ is the density, $A$ is the cross-sectional area.

By (2), (3) the simultaneous angular frequency formula is:

$\Omega^{2}=\frac{\int_{0}^{l} E I\left(\frac{\partial^{2} y_{0}}{\partial x^{2}}\right)^{2} d x}{\int_{0}^{l} \rho A y_{0}^{2} d x}$

Dynamic frequency calculation, often need to know the modal function of $Y_{0}$ cantilever beam, however accurate modal function is unknown, calculate the vibration frequency on the basis of the modal function of setting a reasonable boundary condition can experience.

$T_{\max }=U_{\max }+U_{\sigma \max }$

In the type: $T_{\max }$ is the maximum kinetic energy, $U_{\max }$ is the work of elastic force, $U_{w \text { Max }}$ is to overcome the centrifugal force.

The dynamic frequency formula for the cantilever beam in space is obtained by the Rayleigh energy method.

$\Omega^{2}=\frac{\int_{0}^{l} E I\left(\frac{\partial^{2} y_{0}}{\partial x^{2}}\right)^{2} d x}{\int_{0}^{l} \rho A y_{0}^{2} d x}+\frac{\rho \omega^{2} \int_{0}^{l} A\left(x+x_{0}\right)\left[\int_{0}^{r}\left(\frac{d y_{0}}{d x}\right)^{2} d x\right] d x}{\int_{0}^{l} \rho A y_{0}^{2} d x}$

In the type: $\omega$ is the angular velocity, $x$ is means to the center shaft distance.

To the left of the above type static frequency is $\Omega_{s}$, in order to better identify the static frequency and centrifugal force of the vibration frequency, the (6) can be changed to:

$\Omega_{d}^{2}=\Omega_{s}^{2}+\Omega_{0}^{2}$

By $f=2 \pi / \Omega$ shows that the dynamic frequency formula:

$f_{d}^{2}=f_{s}^{2}+f_{0}^{2}$

This paper uses SHELL181 unit with cantilever beam structure as the research object [5]. high is $8 m$, width is $0.12 m$, thickness is $0.2 m$, elastic modulus is $2.15 e^{11} p a$, Poisson's ratio is 0.31 , density is $7800 \mathrm{~kg} / \mathrm{m}^{3}$, load and boundary conditions: one end fixed, one end free rotation around the $Z$ axis, the speed of $n=360 \mathrm{rad} / \mathrm{min}$.

This paper mainly lists five order before blade frequency of modal frequency and theoretical calculation of the comparative analysis, such as table 1.

Tab.1 Static and Dynamic Calculation Results

\begin{tabular}{|c|c|c|c|c|c|c|}
\hline & Order & $\begin{array}{c}\text { First } \\
\text { order }\end{array}$ & $\begin{array}{c}\text { Second } \\
\text { order }\end{array}$ & $\begin{array}{c}\text { Third } \\
\text { order }\end{array}$ & $\begin{array}{c}\text { Fourth } \\
\text { order }\end{array}$ & $\begin{array}{c}\text { Fifth } \\
\text { order }\end{array}$ \\
\hline $\begin{array}{c}\text { theoretical } \\
\text { value }\end{array}$ & $\begin{array}{c}\text { Static frequency } \\
(\mathrm{Hz})\end{array}$ & 1.0406 & 6.5172 & 6.7781 & 18.258 & 35.745 \\
\cline { 2 - 7 } & $\begin{array}{c}\text { Dynamic } \\
\text { frequency } \\
(\mathrm{Hz})\end{array}$ & 1.5113 & 6.7925 & 7.0014 & 18.738 & 36.262 \\
\hline \multirow{2}{*}{$\begin{array}{c}\text { ANSYS } \\
\text { value }\end{array}$} & $\begin{array}{c}\text { Static frequency } \\
(\mathrm{Hz})\end{array}$ & 1.0400 & 6.5165 & 6.7773 & 18.242 & 35.735 \\
\cline { 2 - 7 } & $\begin{array}{c}\text { Dynamic } \\
\text { frequency } \\
(\mathrm{Hz})\end{array}$ & 1.5076 & 6.7916 & 7.0002 & 18.729 & 36.240 \\
\hline
\end{tabular}

The table 1 shows that the ANSYS software analysis of the blade frequency and the theoretical value is very close, dynamic and static frequency error is less than $1 \%$, ANSYS numerical results to meet the needs of the analysis. The real variable section (variable thickness and width) blade adopts analytic method, which is difficult to solve. Therefore, the finite element method is used to analyze the real blades, which not only improves the accuracy, but also improves the efficiency. In this paper, the first order torsion of different position and depth of the blade is analyzed. 


\section{Blade Model Building}

In this paper, Blade geometry model is figure 1 , the height of the blade root is $0.5 \mathrm{~m}$, and the height of $L$ is $8 \mathrm{~m}$. The blade material is made of an orthotropic glass fiber reinforced plastic composite material, which parameters in the normal temperature are as follows:Tab.2[6]. Table $E_{1}$ indicates spanwise elastic modulus, $E_{2}$ is the radial elastic modulus, $G_{1}$ is the shear modulus ,NUXY is the Poisson's ratio, $\rho$ is the density. The distance from the root of the crack is $L_{1}$, which depth is $a$. The geometric model is shown in Figure 1.

Tab.2 Material Parameter

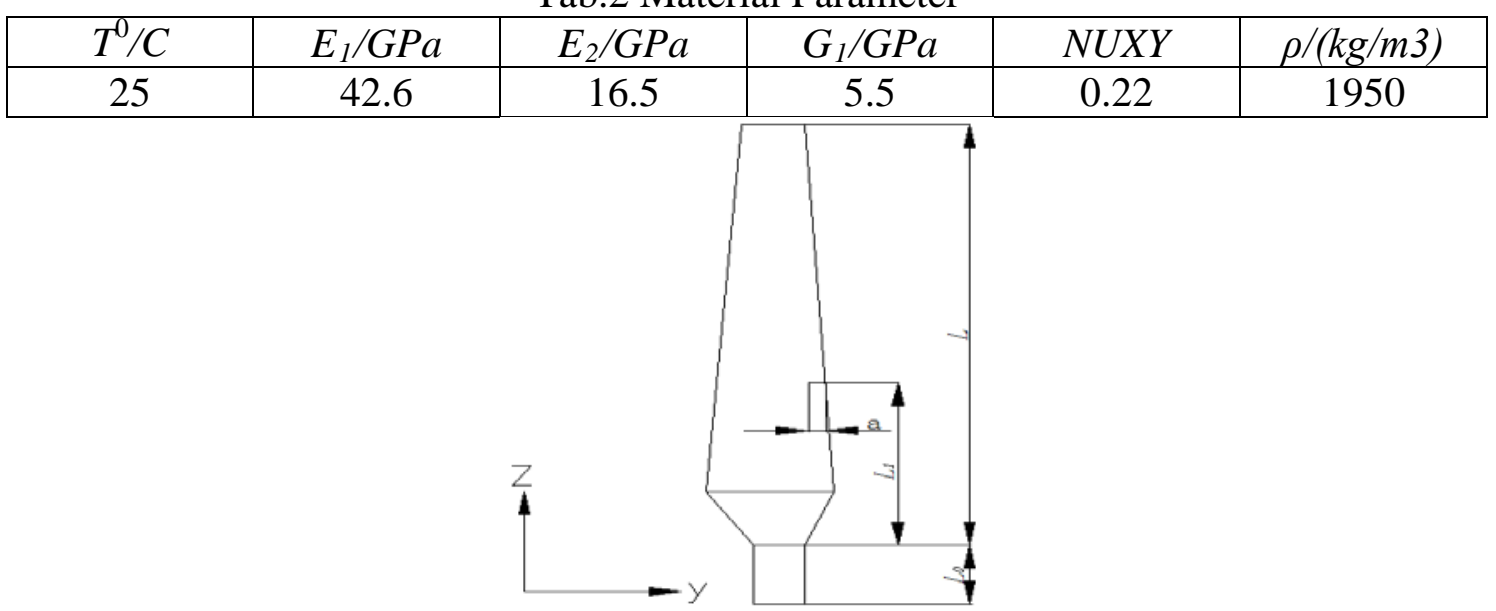

Fig.1 Blade Geometry Model

\section{Result analysis}

The crack depth at the distance of the root $L_{1}=1 / 9$ is $a=1 \mathrm{~mm}, 2 \mathrm{~mm}, 3 \mathrm{~mm}, 4 \mathrm{~mm}, 5 \mathrm{~mm}$ and other different depth of crack, $Y$ direction of the first order dynamic frequency at different speeds, as shown in figure 2.

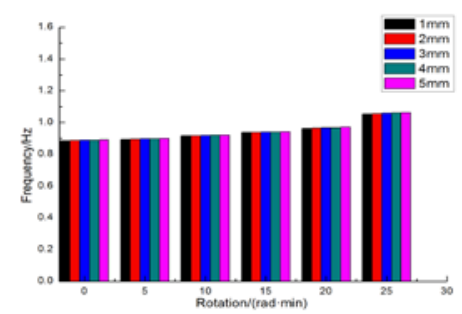

Fig.2 Relationship Between the First Order Dynamic Frequency and Rotational Speed of $Y$ direction

By Fig. 2, the same crack depth with different frequency under different speeds. When the fan rotates, it is subjected to the action of centrifugal force acting on the crack . The increase of the stiffness of the blade caused the frequency of the crack is also gradually improved and a curve with increasing frequency and increasing speed is formed. The depth of the crack at the same speed of change is not obvious. The variation trends of different crack depths at different speeds are similar to those of the same crack at different speeds.

Crack at different depths in the same position of blade, $X$ direction of the first order dynamic frequency at different speeds of the relationship, as shown in Fig. 5. 


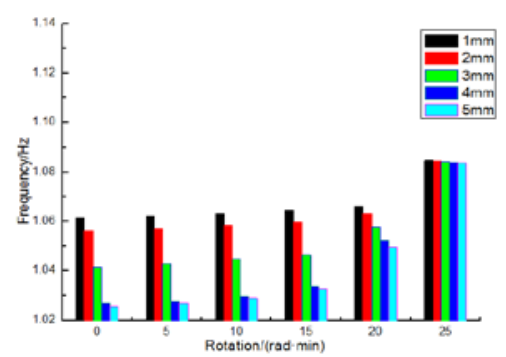

Fig.3 Relationship Between the First Order Dynamic Frequency and Rotational Speed of $X$ direction

By figure 2-3 shows that the vibration of blade in $X$ direction is more complicated than that in $Y$ direction. In Figure 3, In the same condition of rotating speed, the deeper the crack is, the smaller the corresponding frequency of $X$. The depth of the blade with smaller crack depth is deeper than the deep blade with high natural frequency. The $X$ direction of different crack depth first order the dynamic frequency is as speed increases, the dynamic frequency frequency is also rising.

There is a different depth crack at the distance of the blade root $L_{1}=1 / 9$. The speed and crack depth are not the same as the first order torsion frequency of the blade through the modal analysis.

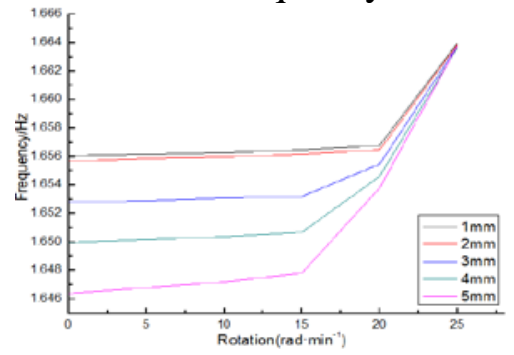

Fig.4 First Order Torsional Frequency Curves at Different Speeds

Fig.4 shows the $0-15 \mathrm{rad} / \mathrm{min}^{-1}$ speed range of different crack depth of the crack, with increasing speed, frequency increases slowly; When crack depth of $3 \mathrm{~mm}, 4 \mathrm{~mm}, 5 \mathrm{~mm}$, The rotational speed is higher than $15 \mathrm{rad} / \mathrm{min}^{-1}$, the first order torsion frequency increase; The curves of different depth crack change law curve are similar.

There is a relative position of $L_{1} / L=0,1 / 9,1 / 4,1 / 3,4 / 9,5 / 9,2 / 3,7 / 9$ different cracks.Through multiple modeling, the relative position of the first order dynamic frequency and crack of the crack blade is analyzed, as shown in Fig.5 and 6.

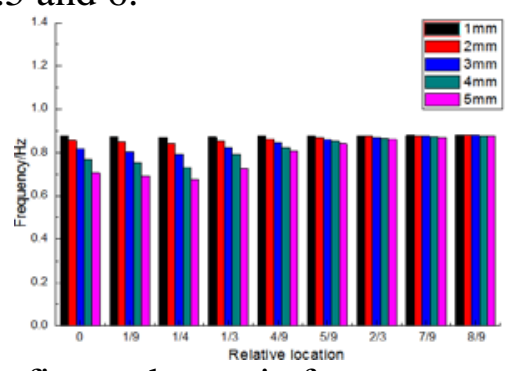

Fig.5 The relationship between the first order static frequency and the relative position of the crack

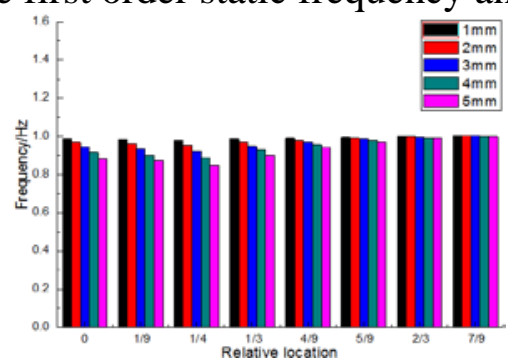

Fig.6 The relationship between the first order dynamic frequency and the relative position of the crack

From Fig.5, 6 show that the blade crack with the crack location from root to tip changes,the first order static and dynamic frequency of blade decreases and then increases. For the blade with different crack depth, the relative position of $1 / 4$ is the lowest. It is indicated that the $1 / 4$ is prone to 
failure at the relative position, the blade should be focused on the area once the crack failure occurs. Along with the crack position away from the blade root, the rate of change of the blade's static and dynamic frequency is also slow down.

Cracks at different depths in the $\mathrm{L} 1=1 / 9$ of the root of the leaves, the relationship between the first order dynamic frequency and the crack depth of the blade is shown in Fig.7.

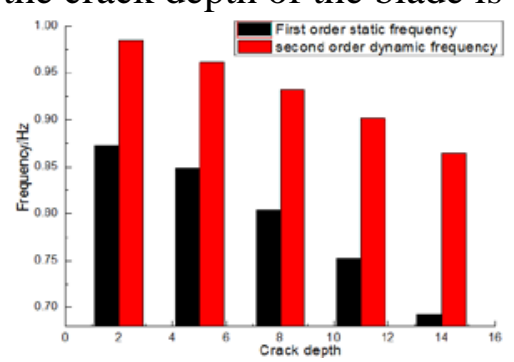

Fig. 7 Relationship between crack depth and first order dynamic frequency

From Fig.7, the static frequency of the same crack depth crack blade is lower than the frequency of the dynamic frequency; when the crack depth is extended, the blade dynamic and static frequency are decreased, and the rate of decline is more and more fast. The gradual increase of the crack depth greatly weakens the structural stiffness, reduces the natural frequency of the blade, but has no obvious effect on the quality.

\section{Conclusion}

In this paper, an analytical method is used to derive the dynamic equation of the blade, the finite element method is used to analyze the dynamic frequency of the blade,the results of comparative analysis and finite element analysis show that the finite element analysis method is effective and correct. Influence on the variation of frequency of crack direction and speed are similar, The natural frequency increases with the increase of the velocity and the change of the crack location, blade dynamic and static frequency changes showed a trend of decreased first and then increased, the crack depth, the lower the frequency of leaf movement.

\section{Acknowledgement}

In this paper, Major projects of Henan Province Foundation and Advanced Technology Research Project(Project No.152300410083) .The Henan University of Science and Technology Senior Project Fund (Project No.2015GJB006).

\section{References}

[1] Luping Li,Mangmang Li,Feng Ji,et al.Application of vibration detection technology in the fault monitoring of wind turbine blades[J].Journal of Engineering for Thermal Energy and Power,2013,28(2):207-212.

[2] Xiangbin Chen,Baocang Sun.Modal characteristics analysis of small wind turbine blades[J].Energy Research and Information,2013,29(4):192-200.

[3] Bonan Fan,Yubo Zhang,Haidou Wang,et al.Effects of crack parameters on the vibration characteristics of low order flexural vibration of blades[J].Surface Technology,2015,44(9):96-101.

[4] Shijun Zhou,Degui Liu.Finite element analysis of dynamic characteristics of arbitrary shape plate considering initial load effect[J].Journal of Vibration and Shock,2014,(12):15-23.

[5] Baoqing Yang,Liming Zhang,Xiaokun Wang.Deflection formula of cantilever beam with variable cross section in N section[J].Anhui Architecture,2012(6):60.

[6] Deyuan Li,Zhiquan Ye,Yan Chen,et al.Fatigue life analysis of glass fiber reinforced plastic blades[J].Acta Energiae Solaris Sinica,2004,25(5):592-598. 